\title{
Bunje (Novo Selo, Croatie). Chronique des activités archéologiques, 2019
}

Emmanuel Botte, Audrey Bertrand, Jere Drpić, Kristina Jelinčić, Bastien Lemaire, Nicolas Leys, Alexia Rosak, Florian Huvet, Antoine Boisson et Léo Cagnard

\section{(2) OpenEdition}

\section{Journals}

\section{Édition électronique}

URL : http://journals.openedition.org/cefr/4606

DOI : $10.4000 /$ cefr.4606

ISSN : 2282-5703

Éditeur

École française de Rome

\section{Référence électronique}

Emmanuel Botte, Audrey Bertrand, Jere Drpić, Kristina Jelinčić, Bastien Lemaire, Nicolas Leys, Alexia Rosak, Florian Huvet, Antoine Boisson et Léo Cagnard, « Bunje (Novo Selo, Croatie). Chronique des activités archéologiques, 2019 », Chronique des activités archéologiques de l'École française de Rome [En ligne], Balkans, mis en ligne le 16 juillet 2020, consulté le 17 juillet 2020. URL : http:// journals.openedition.org/cefr/4606; DOI : https://doi.org/10.4000/cefr.4606

Ce document a été généré automatiquement le 17 juillet 2020.

(c) École française de Rome 


\title{
Bunje (Novo Selo, Croatie). Chronique des activités archéologiques, 2019
}

\author{
Emmanuel Botte, Audrey Bertrand, Jere Drpić, Kristina Jelinčić, Bastien \\ Lemaire, Nicolas Leys, Alexia Rosak, Florian Huvet, Antoine Boisson et Léo \\ Cagnard
}

\section{Introduction}

1 Le chantier de Bunje sur l'île de Brač constitue la première étape d'un programme portant sur les villae de l'Adriatique orientale, conduit par E. Botte (centre Camille Jullian, CNRS), A. Bertrand (Univ. Paris-Est) et K. Jelinčić (Institut Archéologique de Zagreb) et intitulé «Recherches sur l'exploitation économique de la Dalmatie à l'époque romaine (II s. av. J.-C. - II ${ }^{\mathrm{e}} \mathrm{s}$. apr. J.-C.) ». L'intention des auteurs de ce projet est de mesurer, à travers des opérations de fouilles et de prospections, l'impact économique de la présence romaine en Dalmatie ${ }^{1}$.

2 La première campagne menée en 2015 sur le site de Bunje (commune de Novo Selo) sur l'île de Brač (fig. 1) était destinée à obtenir une vision d'ensemble de l'ampleur du site et de l'état de préservation de ses vestiges. Il en était ressorti un site assez étendu (les vestiges couvrant un espace de près de $15000 \mathrm{~m}^{2}$, et la villa couvrant à l'intérieur de celui-ci près de $2500 \mathrm{~m}^{2}$ ) et dont les structures sont dans l'ensemble assez bien conservées, ce qui confirme les observations effectuées lors d'un premier rapport mené par K. Jelinčić en $2012^{2}$. Le site est inscrit au registre des monuments culturels sous le numéro 486, et protégé en tant que tel auprès de la Surintendance de Split par décision du 26 septembre 1979 (sous le numéro 17/41-1979).

Fig. 1 - Carte de situation de l'île de Brač et de la commune de Novo Selo. 


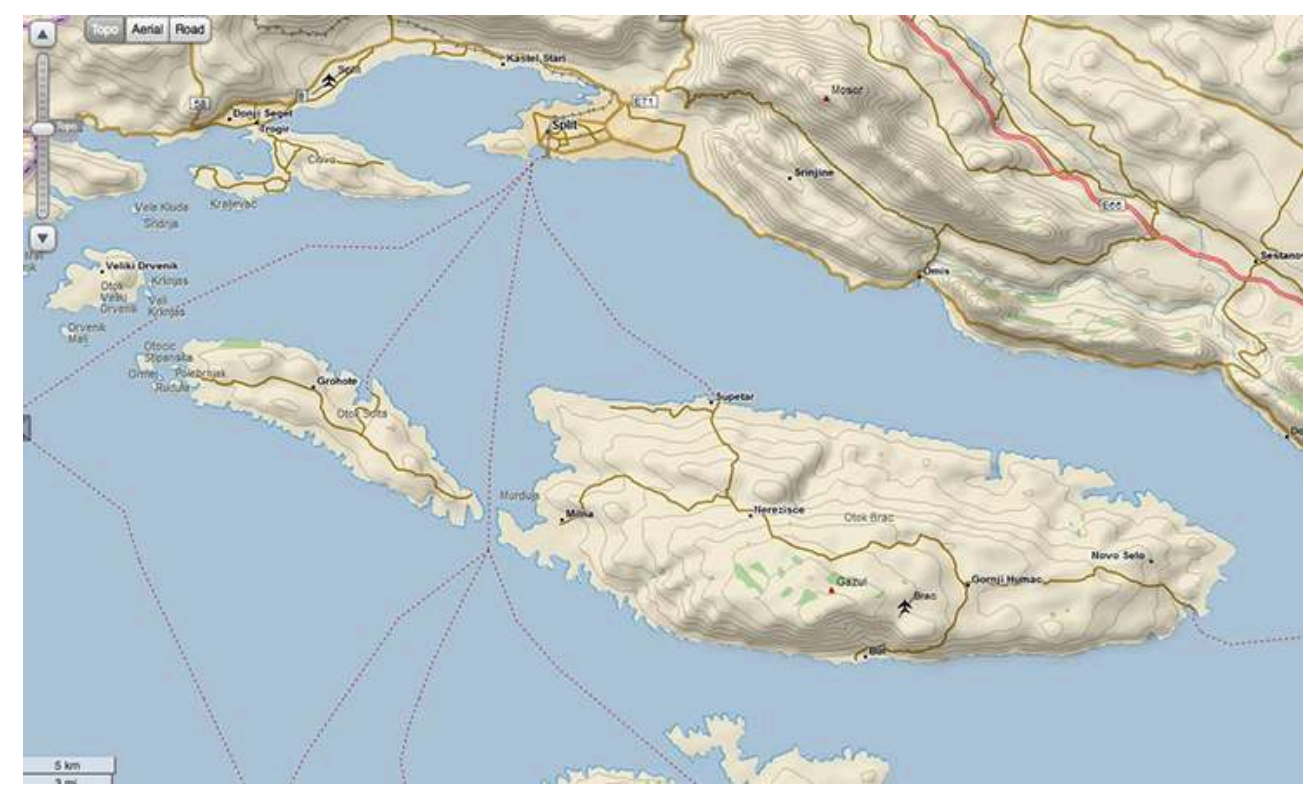

(c) GoogleMaps

Les opérations menées sur ce site s'inscrivent dans une recherche plus large visant à clarifier les mécanismes et la chronologie de l'occupation du territoire de la Dalmatie centrale en amont et en aval de la conquête romaine de ce territoire à la fin de la période tardo-républicaine. Au sein de cette problématique, les fouilles de la villa de Bunje nous permettront de déterminer le cadre historique et environnemental qui accueille cette structure, ainsi que l'organisation de sa production.

4 En 2017 et avril 2018, les campagnes de fouilles nous avaient permis de faire des avancées significatives dans la compréhension du plan de la villa, en faisant apparaitre la partie résidentielle (pars urbana) et la partie productive (pars rustica) de part et d'autre d'une grande cour centrale. Nous avions pu notamment compléter le plan dans les secteurs méridionaux et orientaux, faisant apparaître un bâtiment au sud-est des structures connues, ainsi qu'un pressoir à huile dans un très bon état de conservation.

La campagne d'octobre 2018, concentrée sur le secteur oriental de la pars rustica, n'avait pas donné d'indication claire sur la présence d'un chai, mais nous avait permis de comprendre qu'une seconde terrasse, située en contrebas de celle connue, avait fait l'objet d'une occupation durant l'Antiquité.

\section{Déroulement et objectifs de la campagne de fouilles}

6 La campagne de fouilles s'est déroulée durant le mois d'octobre, sous la direction d'E. Botte (CNRS, Centre Camille Jullian), A. Bertrand (Université Paris-Est Marne-laVallée) et J. Drpić (Institut d'archéologie de Zagreb). L'équipe de fouille était constituée de Nicolas Leys (Sorbonne Université), Bastien Lemaire (Univ. Montpellier), Florian Huvet (Mosaïques Archéologie), Alexia Rosak, Antoine Boisson (INRAP) et Léo Cagnard.

7 La campagne a été financée par l'École française de Rome, le Ministère de l'Europe et des Affaires étrangères, le Centre Camille Jullian, le Ministère de la Culture de Croatie et la municipalité de Selca. Les mobiliers en cours d'étude et ceux considérés comme importants pour la datation et l'histoire du site ont été consignés à l'Institut archéologique de Zagreb. L'étude archéozoologique est réalisée par Siniša Radović 
(Académie des sciences et des arts à Zagreb) ; l'étude anthracologique par Christophe Vaschalde (Université Montpellier 3); l'étude paléobotanique par Margaux Tillier (Université Montpellier 3) ; l'étude micro-morphologique des sols par Cristiano Nicosia (Université de Padoue) ; l'étude du verre par Bartul Siljeg (Institut Archéologique de Zagreb); les études anthropologiques par Mario Novak (Institut d'Anthropologie de Zagreb) ; les études numismatiques par Anja Bertol (Université de Zagreb) ; les analyses chimiques par Nicolas Garnier (Laboratoire Nicolas Garnier). Nicolas Leys, responsable de la photogrammétrie sur le site, a fait une modélisation 3D du site et des principaux blocs de pierre. Les relevés topographiques et les plans ont été réalisés par J. Drpič. Létude du mobilier céramique a été réalisée par Emmanuel Botte et les dessins par Antoine Boisson.

8 Les objectifs de la campagne d'octobre étaient concentrés sur le secteur 6, qui ouvre sur la cour centrale et qui n'avait pas encore été dégagé, et sur la terrasse inférieure (secteur 4) qui a été identifiée à la fin de la campagne précédente (fig. 2-3). L'objectif était notamment de saisir si cette terrasse est contemporaine de la terrasse supérieure et de déterminer la nature de son occupation (productive ou résidentielle).

Fig. 2 - Vue du site par drone au début de la campagne.

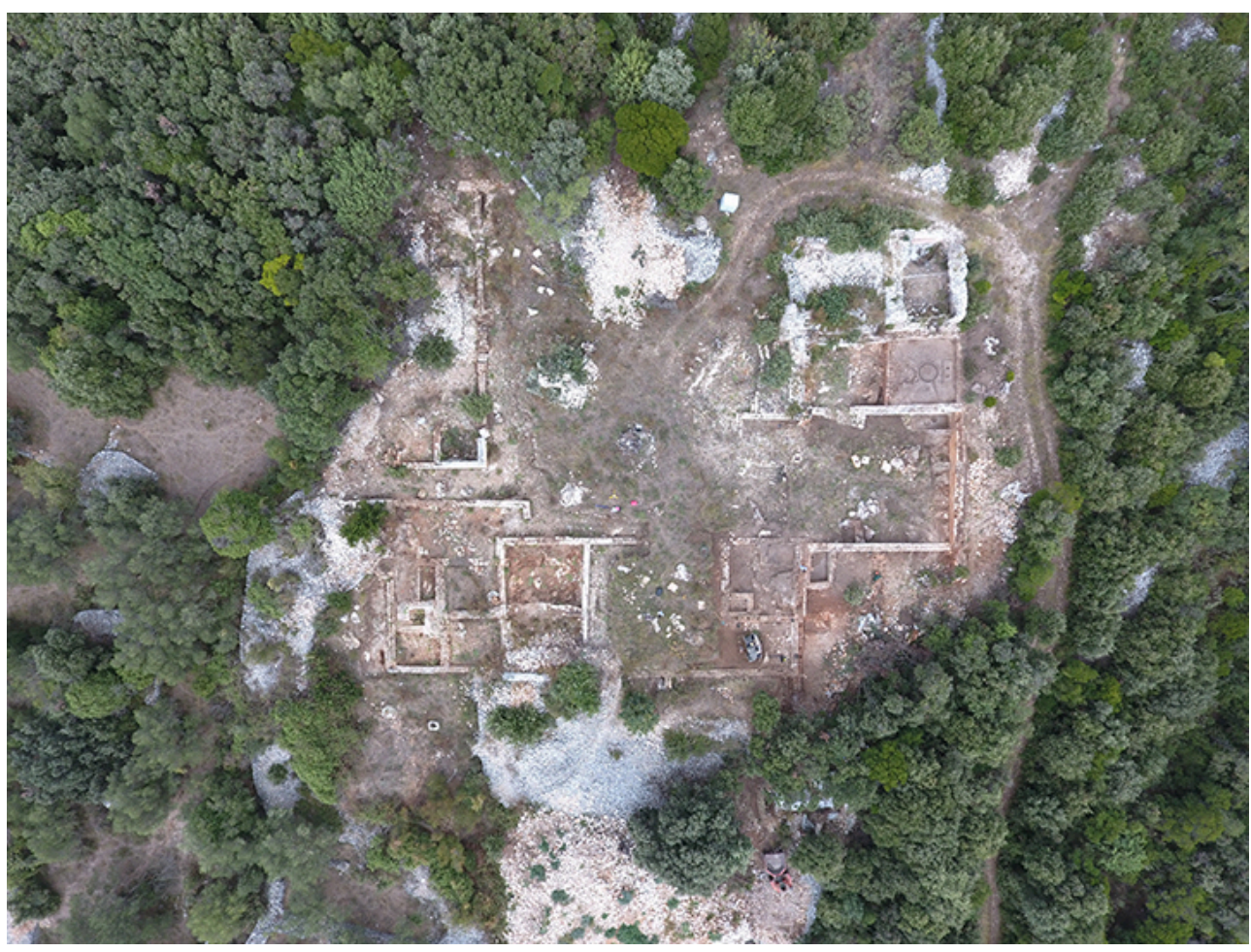

Photo E. Botte.

Fig. 3 - Plan de la villa avec les secteurs fouillés durant cette campagne (situation avant la fouille). 


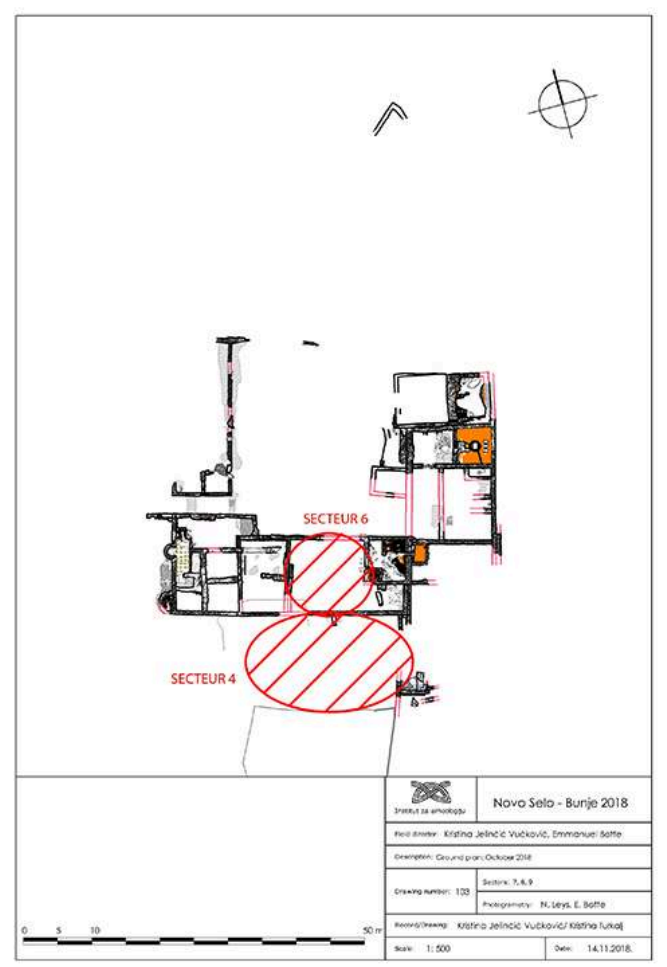

Plan K. Jelinčić.

Une grande partie du travail de cette campagne a consisté, comme c'est désormais l'habitude sur ce site, à démanteler les murs modernes à l'aide d'une pelle mécanique mise à disposition par la mairie de Selca. En effet, le site ayant fait l'objet de cultures agricoles depuis près d'un siècle, les différentes générations ont constitué sur le site de grands amas de pierres (gomila) pouvant atteindre près de $2 \mathrm{~m}$ de hauteur, et qui gênent considérablement la lecture du site dans son ensemble. Une grande partie de ces amas de pierres et du mobilier archéologique qui s'y trouve correspond au démantèlement des structures archéologiques de la villa antique. De ce fait, le démontage de ces structures est réalisé systématiquement en présence de plusieurs membres de l'équipe, le mobilier conservé et les pierres travaillées mises de côté en vue de la restauration du site et de sa présentation au public lorsque les fouilles seront achevées. Cette année, l'ensemble du secteur 4 était couvert de ces amas de pierres.

\section{Résultats}

\section{Secteur 6 (fig. 4)}

Fig. 4 - Plan du secteur 6 à la fin de la campagne. 


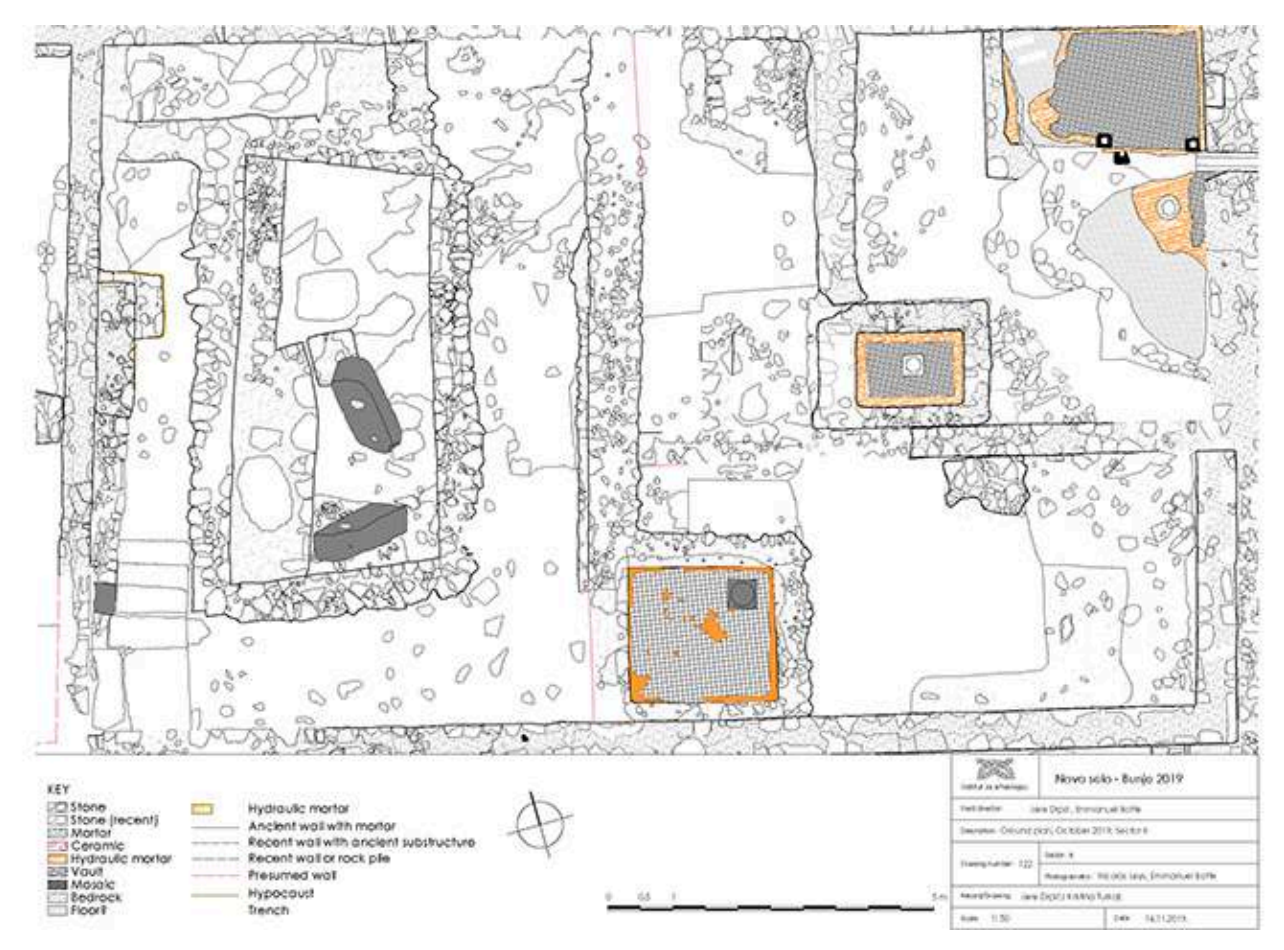

Plan J. Drpič. espace dans lequel se trouvaient un fouloir et un bassin. Ces structures représentaient moins de la moitié de la superficie du secteur 6, et il nous fallait donc comprendre l'organisation de cet espace ouvrant sur la cour centrale.

11 Sous une couche d'humus relativement importante, car la zone était mise en culture pour de la vigne jusque récemment, un grand niveau de destruction et/ou abandon est apparu (US 6049 et 6050).

Cette couche recouvrait une fosse bâtie dans laquelle deux grands piliers en calcaire étaient encore en place. Mesurant 2,20 m de hauteur, $0,60 \mathrm{~m}$ de large et $0,40 \mathrm{~m}$ de profondeur, ils correspondent aux blocs de treuil d'un grand pressoir à levier qui a malheureusement disparu. Le pressoir étant en général surélevé, il a dû être détruit lorsque les structures ont été arasées pour aménager une surface cultivable. De ce grand pressoir ne subsiste donc que la fosse de manipulation du levier et un bassin situé en contrebas, sur le flanc sud de l'espace où devait se trouver le pressoir (fig. 5-7). Ce bassin, comme tous ceux que nous avons mis au jour sur le site, a reçu un sol en mosaïque de tesselles blanches de calcaire. Dans ce sol est insérée une coupelle de vidange en calcaire, exactement comme le bassin voisin BS 6002. Comme se pose à chaque fois la question de la production de l'huile ou du vin sur le site, nous avons procédé à une série de prélèvements, d'une part sur la coupelle de vidange en calcaire et d'autre part sur les boudins d'étanchéité du bassin réalisés en béton de tuileau. Ils ont été confiés au Laboratoire Nicolas Garnier pour la réalisation d'analyses afin de déterminer si les marqueurs chimiques présents sont plutôt caractéristiques du jus de raisin ou des acides oléiques caractéristiques de l'huile d'olive, ou bien des deux comme cela est apparu sur d'autres structures de production sur le site. 
Fig. 5 - Vue depuis l'ouest de la fosse abritant les deux blocs de treuil et le bassin au-delà de la fosse.

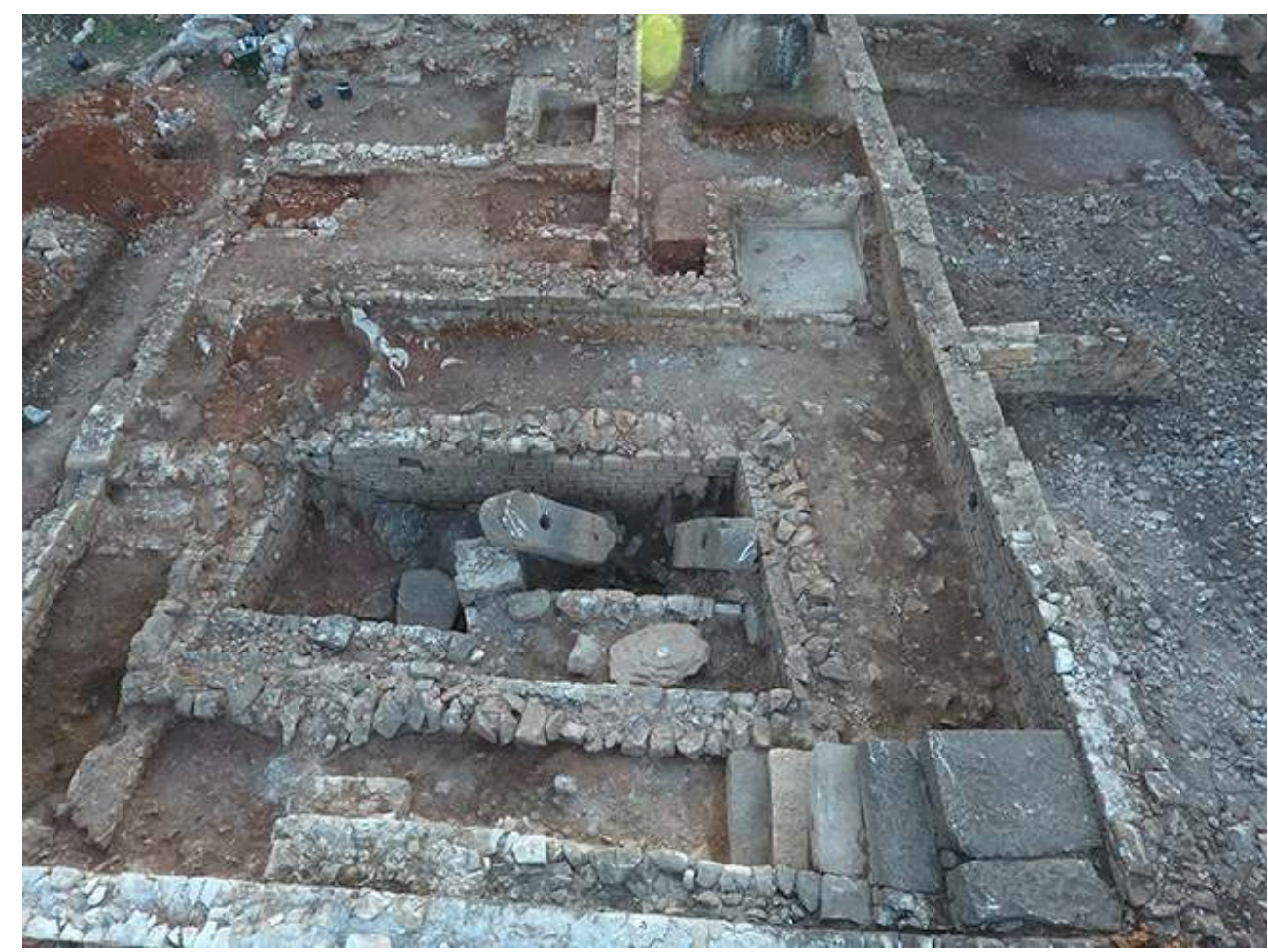

Photo E. Botte.

Fig. 6 - Vue depuis le Nord-Est des blocs de treuil du pressoir.

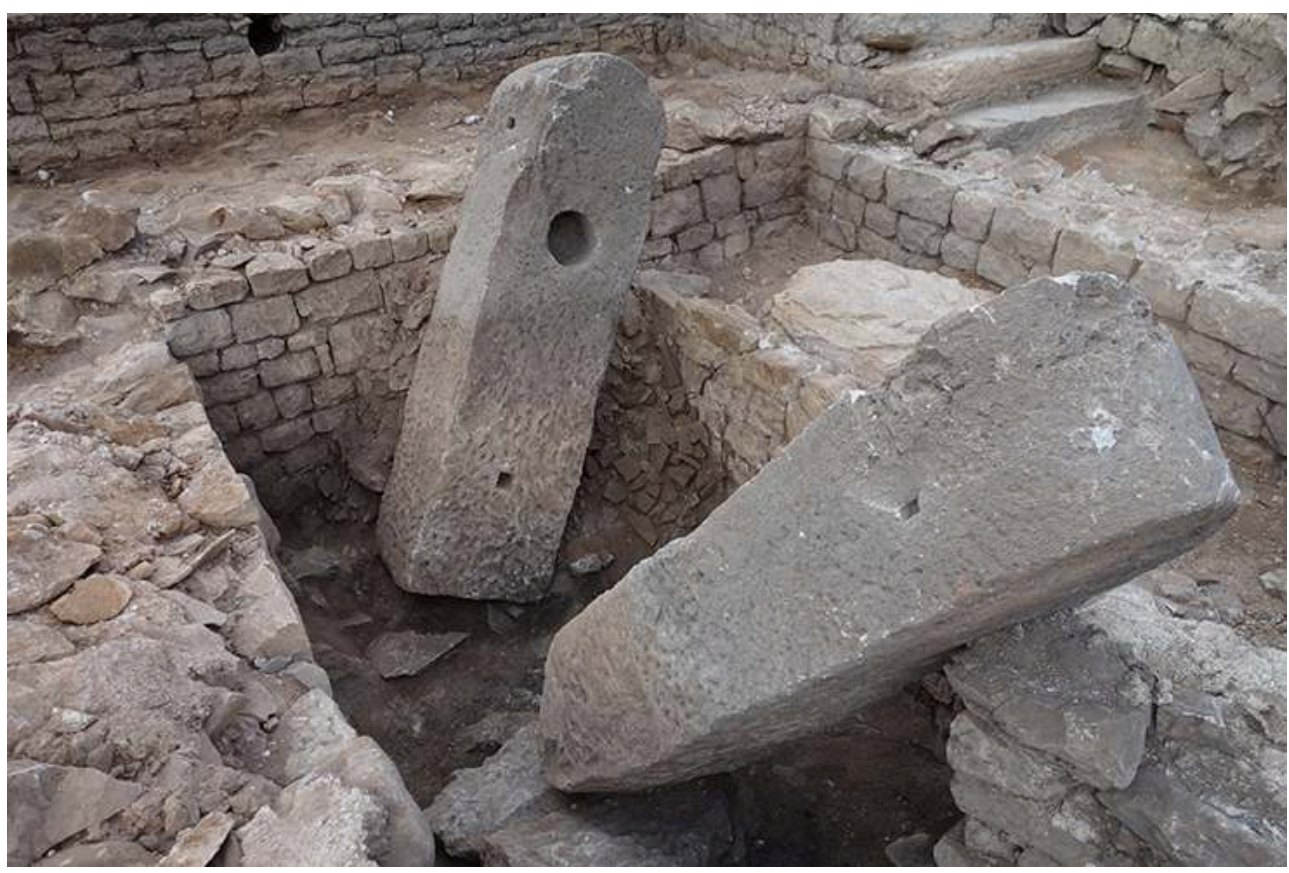

Photo E. Botte. 
Fig. 7 - Vue du bassin récoltant le vin ou l'huile du pressoir aujourd'hui disparu.

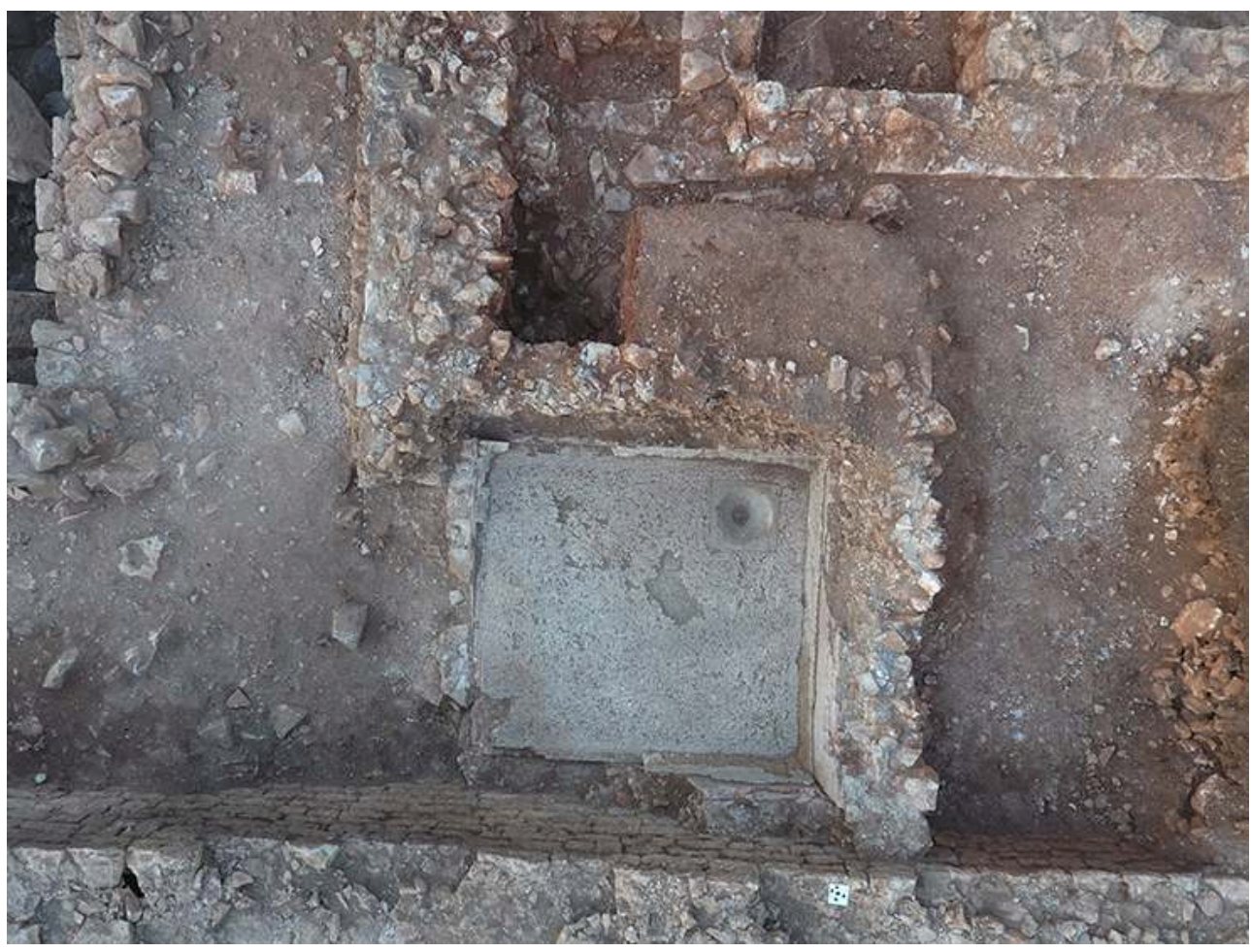

Photo E. Botte.

13 La destination du pressoir, au vu des éléments qui nous sont parvenus, nous oriente plutôt vers la production d'huile d'olive que de vin. D'une part, la présence dans ce même espace d'un moulin (trapetum) destiné à écraser les olives avant de les amener au pressoir est un élément fort en ce sens. D'ailleurs son emplacement au sein de cet espace, au plus près de la zone surélevée où devait se trouvait le pressoir, est également un élément en la faveur de cette destination. D'autre part, les autres sites où une fosse abritant deux grands blocs de treuil a été découverte sont systématiquement liés à la production d'huile. C'est notamment le cas sur le site de Kupinovic sur l'île voisine de Hvar (l'antique Pharos), où des blocs de mêmes dimensions et disposés de la même manière ont été découverts (fig. 8).

Fig. 8 - Vue de la fosse abritant les blocs de treuil de pressoir sur le site de Kupinovik, île de Hvar. 


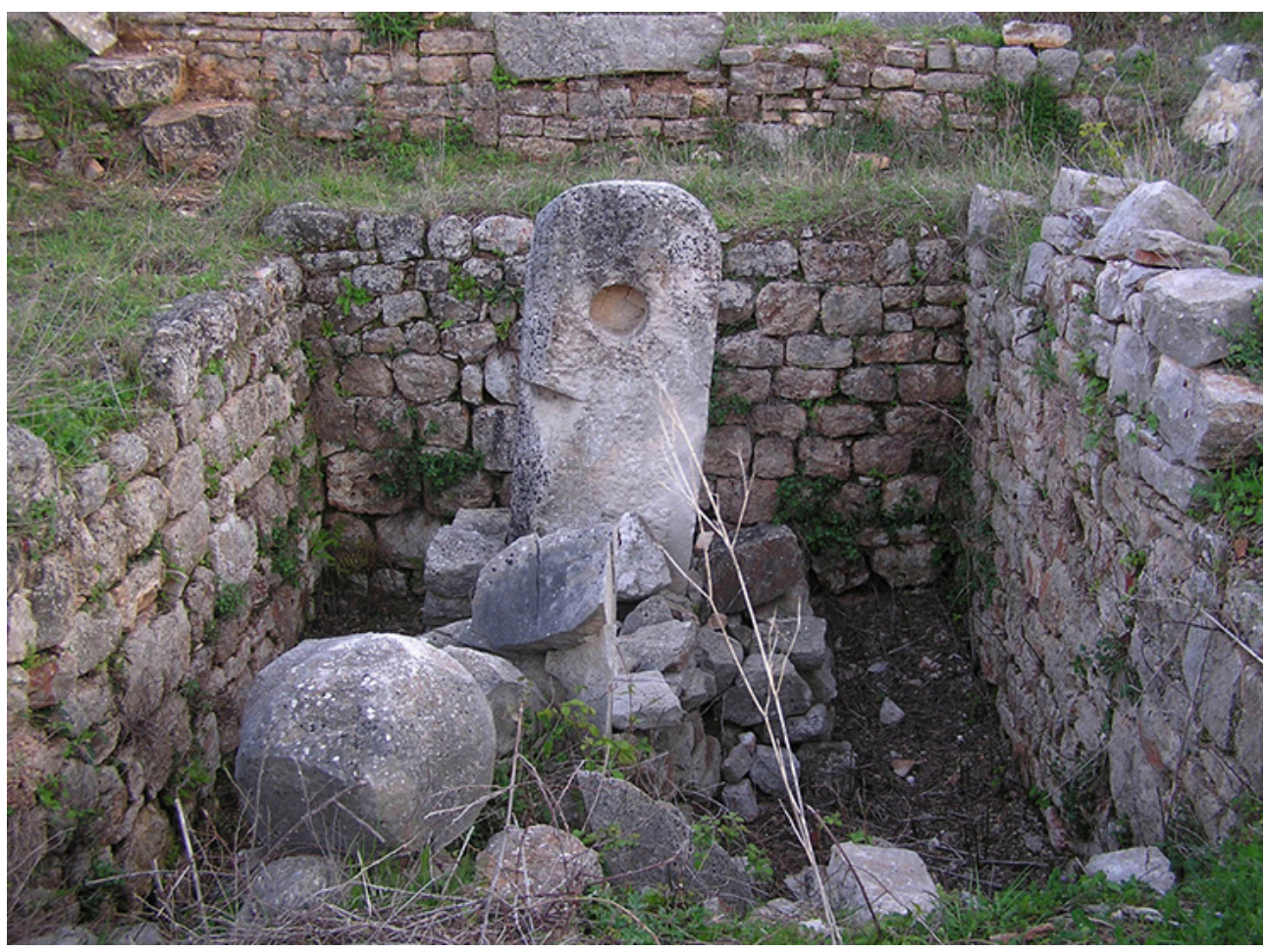

Photo Mladen Plančić.

14 Le mobilier archéologique composant le comblement de la fosse des blocs de treuil nous a apporté de nombreuses informations sur sa nature et sa chronologie (US 6050, 6073 et 6074). Il comporte beaucoup de fragments d'enduits peints (fig. 9), des cailloux mais pas de blocs, une grande quantité de tuiles (tegulae et imbrices), beaucoup de faune et de mobilier céramique (fig. 10). Enfin plusieurs clous en fer complètent cet ensemble. La fourchette chronologique fournie par le mobilier céramique, essentiellement composé d'amphores africaines et orientales, ainsi que de vaisselle en sigillée elle aussi d'origines africaine et orientale, peut être établie entre la seconde moitié $\mathrm{du} \mathrm{V}^{\mathrm{e}}$ siècle et le début $\mathrm{du} \mathrm{VI} \mathrm{VI}^{\mathrm{e}}$ siècle de notre ère. La composition de l'ensemble de ce mobilier nous incite à considérer qu'il correspond au démantèlement de la maison afin de récupérer des matériaux de construction. Cela expliquerait l'absence de moellons travaillés, récupérés systématiquement, ainsi que celle de tuiles complètes, qui ont dû elles aussi être récupérées. Cet ensemble est le premier que nous trouvons en place et qui permet de caler de manière à peu près certaine la période d'abandon, survenue forcément quelques années ou décennies plus tôt, et l'intense phase de récupération des matériaux de la villa.

Fig. 9 - Vue des fragments d'enduits peints découverts dans les niveaux de destruction. 


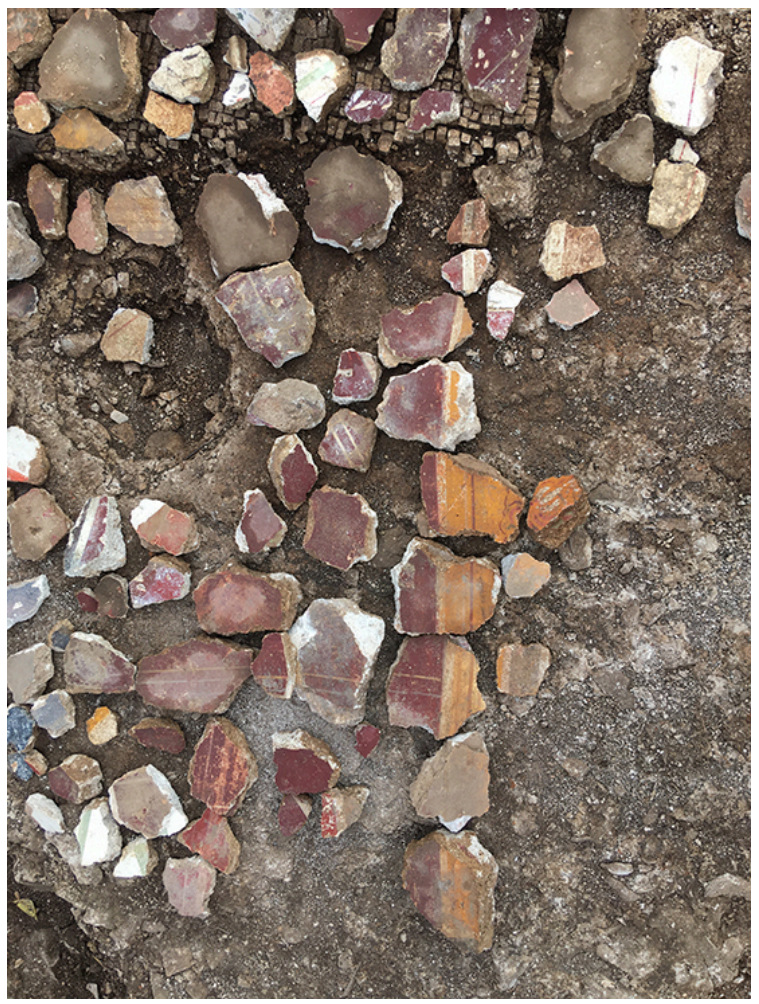

Photo E. Botte.

Fig. 10 - Lampe de production africaine datée du Ve siècle de notre ère.

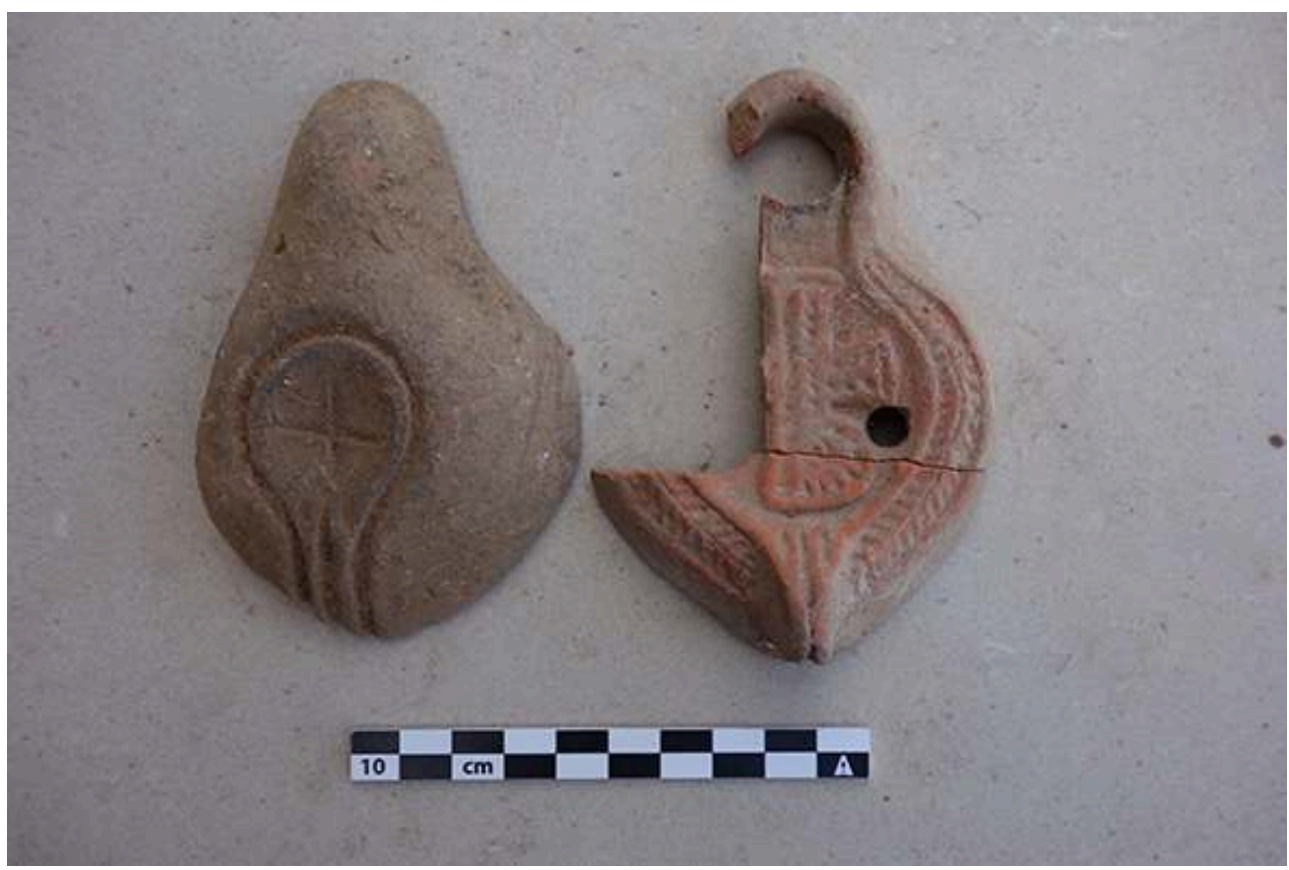

Photo E. Botte.

Dans ce secteur, nous avons pu également mettre au jour, dans l'angle nord-ouest et sous l'espace occupé par la plateforme du pressoir disparu, des niveaux et structures appartenant aux phases antérieures, qu'il faut probablement rattacher à la première 
installation (fig. 11-12). Dans l'angle nord-ouest du secteur 6, nous avons pu fouiller plusieurs niveaux de sols fonctionnant avec deux murs arasés (MR 6090 et 6091). Le mobilier archéologique qui compose ces niveaux stratigraphiques est clairement le plus ancien que nous ayons découvert en place sur le site puisqu'il est datable du milieu du $\mathrm{I}^{\mathrm{er}}$ siècle de notre ère et de la seconde moitié de ce même siècle. Il est notamment composé de tuiles portant des estampilles d'ateliers impériaux appartenant aux empereurs Claude et Néron (fig. 13-14). On trouve également des gobelets en paroi fine de production adriatique ainsi que des fragments de bouteilles en verre qui semblent provenir des ateliers d'Aquilée et qui sont produites durant la seconde moitié du I ${ }^{\text {er }}$ siècle de notre ère.

Fig. 11 - Vue par drone de l'angle nord-ouest du secteur 6 avec les murs arasés appartenant à une phase antérieure.

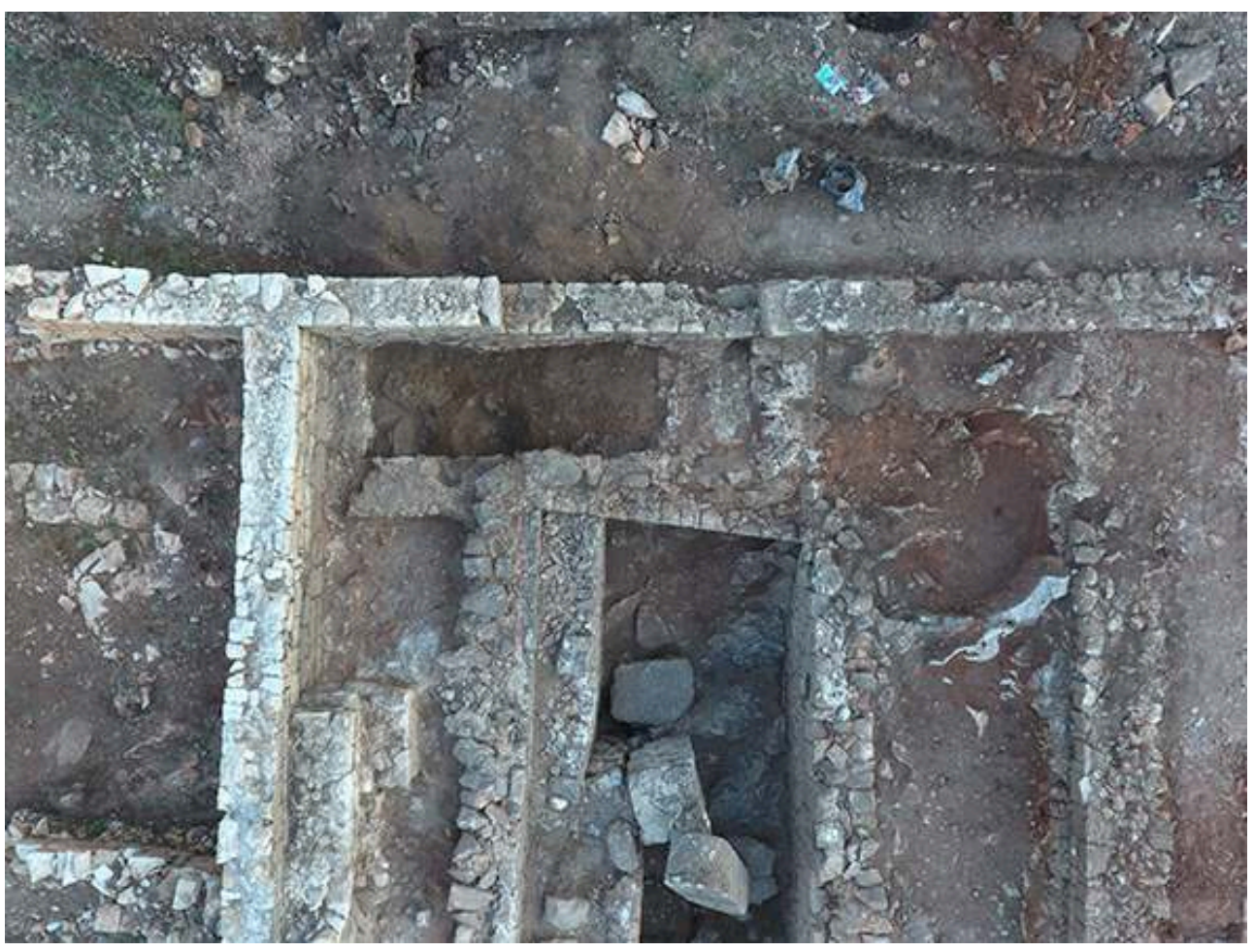

Photo E. Botte

Fig. 12 - Vue depuis l'est des murs arasés MR 6006 et 6078 , comblés par l'US 6078. 


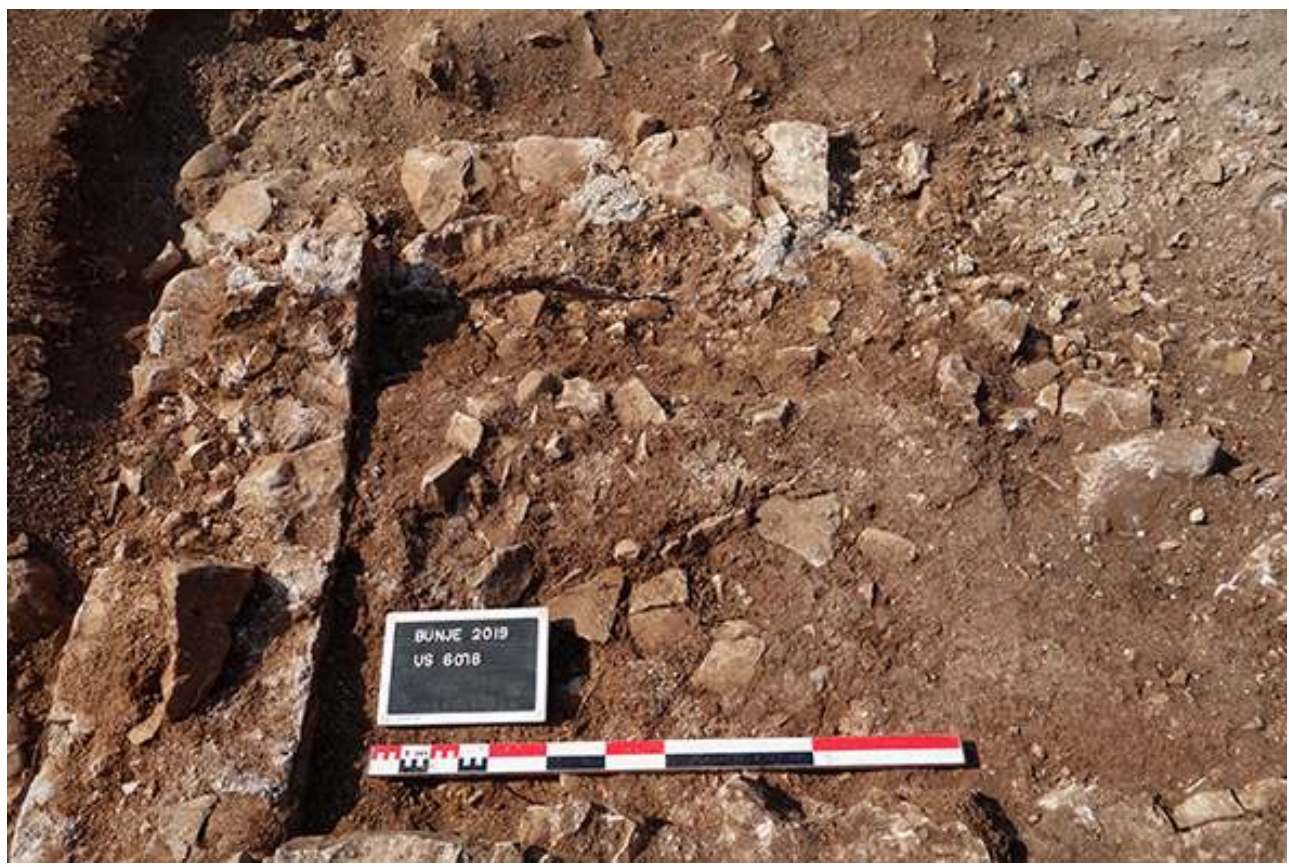

Photo E. Botte.

Fig. 13 - Timbre sur tuile provenant d'un atelier de production propriété de l'empereur Néron (timbre complet : [NERONI]S.CLA.P[AN]).

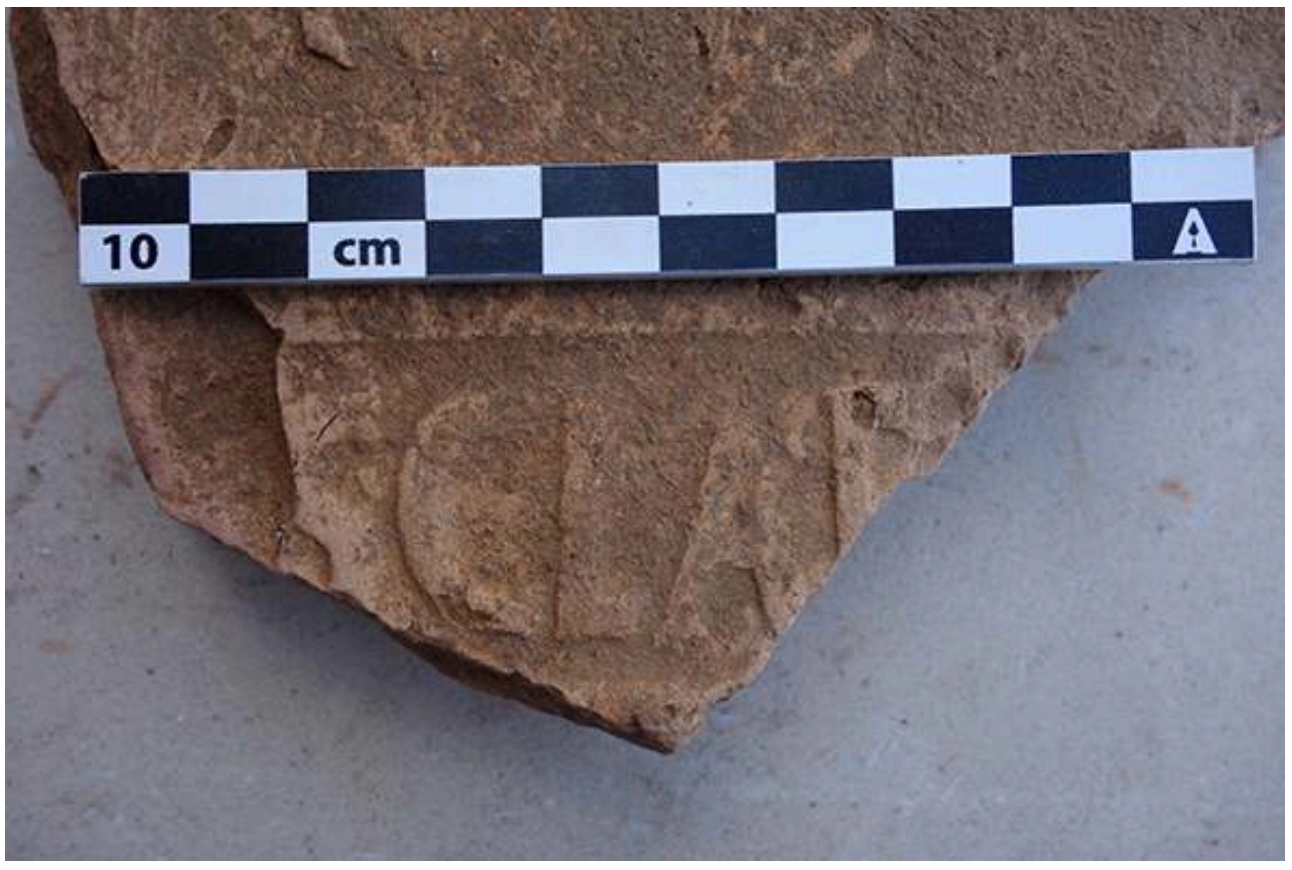

Photo E. Botte.

Fig. 14 - Timbre sur tuile provenant d'un atelier de production propriété de l'empereur Néron (timbre complet : NERO[NIS.CLA.PAN]). 


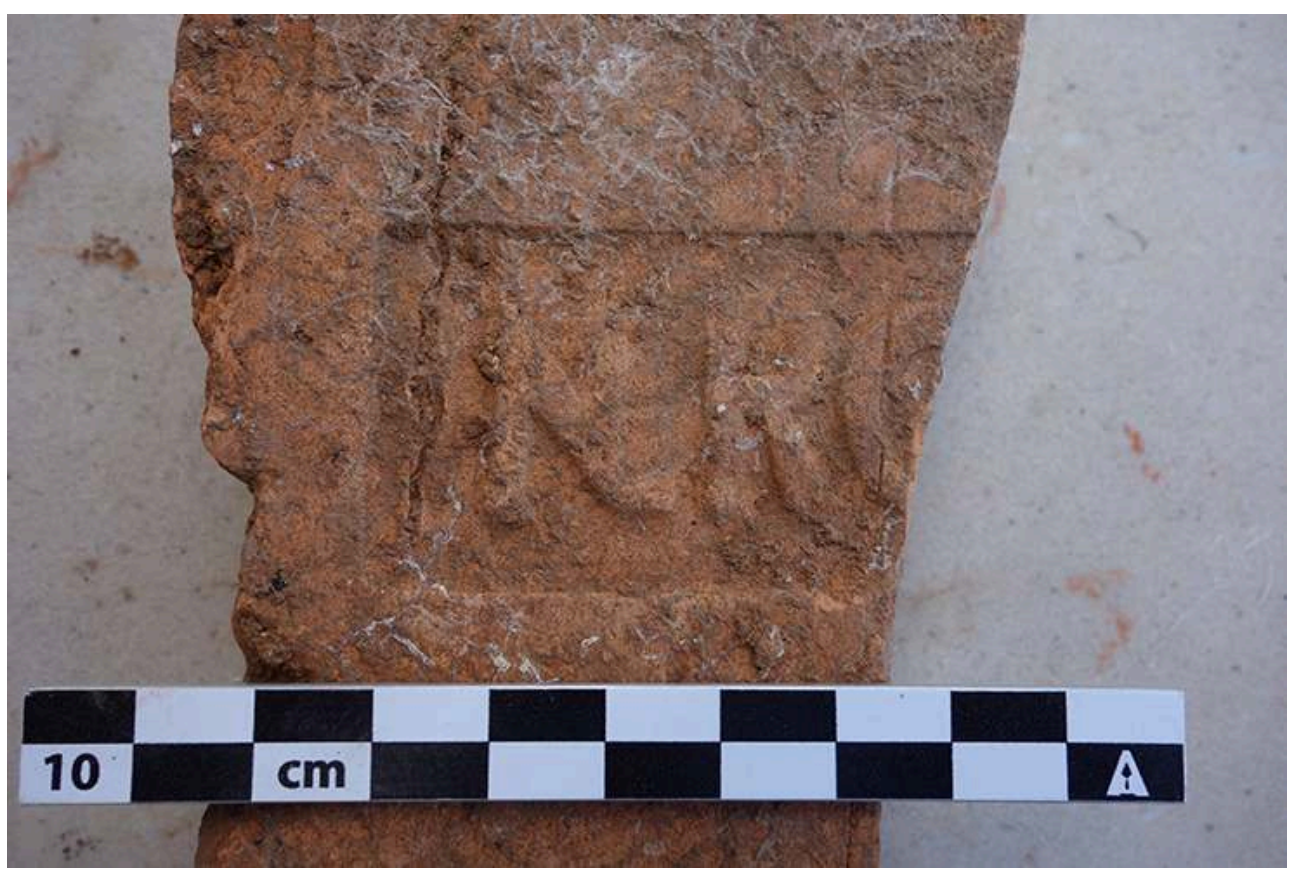

Photo E. Botte.

Dans le niveau US 6078 qui comble l'espace entre les murs arasés MR 6006 et 6078, nous avons découvert peu de mobilier mais celui-ci est riche d'informations car il s'agit de fragments d'un gobelet en céramique à paroi fine ainsi que de céramique dite à vernis rouge pompéien. Cette découverte est très intéressante car elle illustre à la fois un profil chronologique mais également culturel. En effet, ce type de céramique est produit en Campanie du $\mathrm{I}^{\mathrm{er}}$ siècle avant notre ère au $\mathrm{I}^{\mathrm{er}}$ siècle après $\mathrm{J}$.-C., et il est assez peu répandu dans l'Adriatique orientale. Il s'agit de patinae, des plats à cuire revêtus d'un vernis rouge anti-adhérent qui permet d'effectuer des cuissons et notamment des fritures. Or cela constitue est une des caractéristiques des modes de cuisson romains, ce qui nous incite à penser que les occupants de la première installation, comme nous le supposions, pourraient être des colons d'origine italienne.

\section{Secteur 4 (fig. 15)}

Fig. 15 - Plan du secteur 4 à la fin de la campagne. 


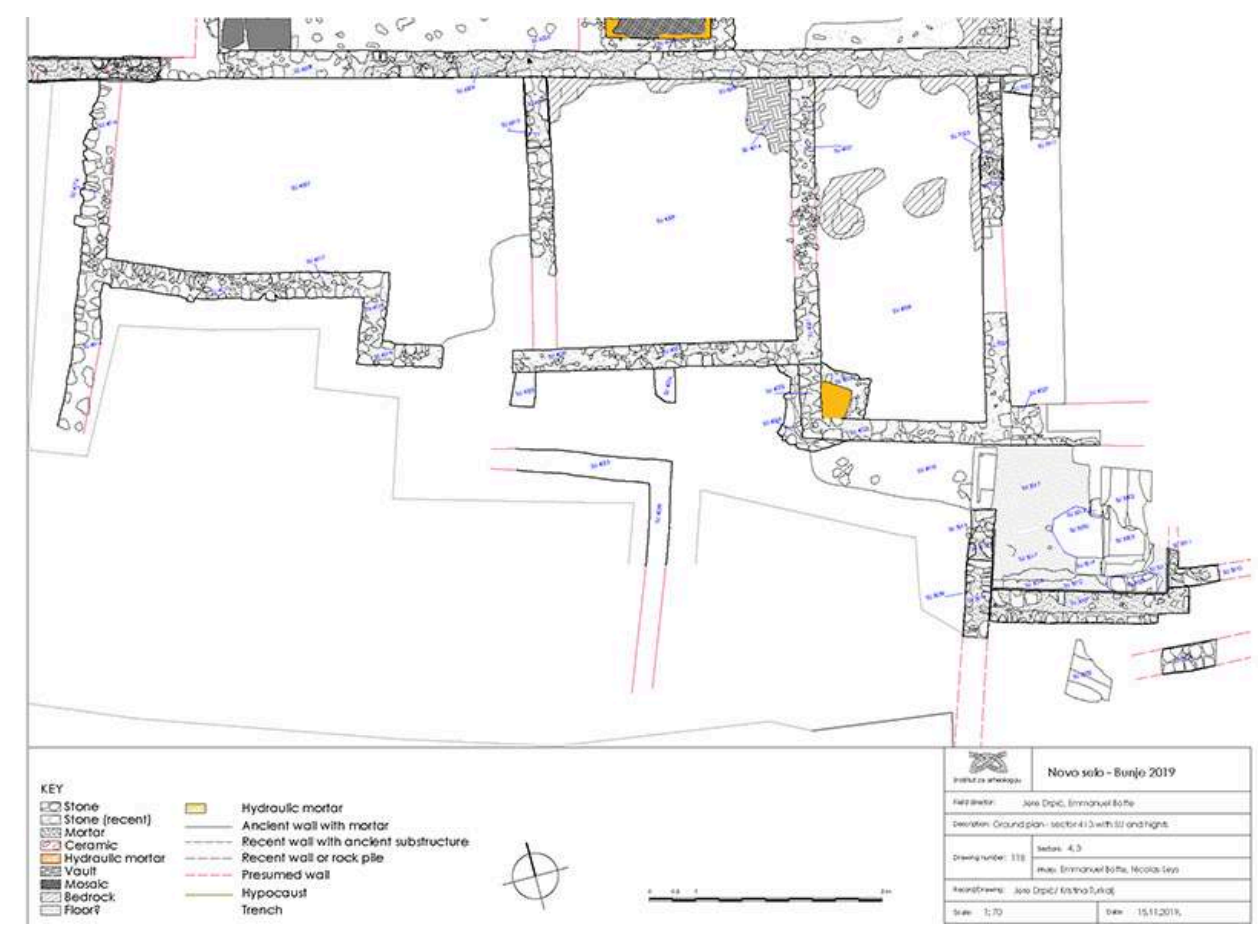

Plan J. Drpič.

Lors de la campagne précédente, nous avions mis au jour le départ d'un mur (MR 7023) appuyé au mur méridional du secteur 6 (MR 6009). Nous en avions alors déduit qu'il existait une terrasse inférieure, dont l'organisation et la chronologie restaient à définir. Nous avons donc consacré une partie de la campagne au dégagement de cette terrasse. Les résultats en sont pour le moment mitigés car nous avons surtout passé l'essentiel du temps à vider les pièces qui étaient remplies de pierres sur parfois plus de deux mètres de hauteur. Il en ressort, dans l'état actuel de conservation, plusieurs pièces presque totalement vides de mobilier, ce qui pour le moment rend la datation de ces espaces délicate (fig. 16). Néanmoins le fait qu'aucune structure de production ou de stockage ne soit présente nous amène à envisager l'hypothèse que cette terrasse ait eu uniquement, ou en tout cas principalement, une vocation résidentielle.

Fig. 16 - Vue depuis le sud des pièces de la terrasse inférieure. 


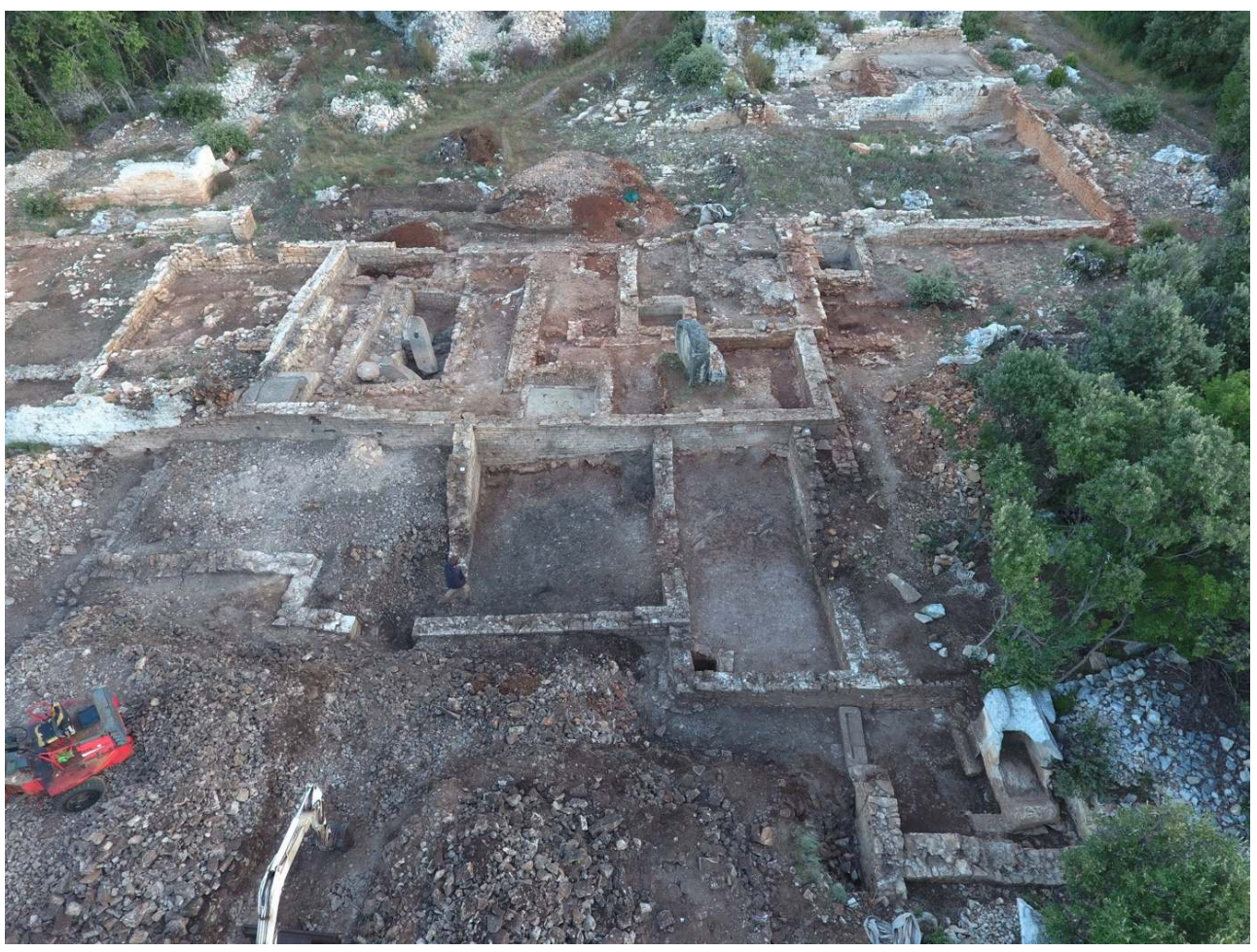

Photo E. Botte.

18 Bien que l'état de conservation de cette terrasse ne soit pas à la hauteur de nos espérances, surtout du point de vue de la stratigraphie, nous avons quand même pu réaliser plusieurs observations. Si nous l'avions déjà supposé lors d'une précédente campagne, il est désormais clair que la pièce dans laquelle se trouve le sarcophage (secteur 3, SP 3004) existe bien avant l'installation de ce dernier. Il apparaît en effet très clairement que le mur MR 3014, qui ferme cet espace à l'ouest, a été coupé pour faire passer le sarcophage, et que l'on a ensuite installé un nouveau seuil, de forme très particulière (fig. 17), et rebouché l'espace entre le seuil et le mur coupé avec un mur (MR 3015) (fig. 18). Dans l'espace qui se trouve devant cette pièce, nous avons suivi un niveau de sol qui semble le dernier en place, caractérisé par un niveau d'éclats de calcaire de moyennes et grandes dimensions, disposés à plat (SL 4010). Nous avons pu suivre ce niveau sur quelques mètres et il semble caractériser le sol d'un espace extérieur, indiquant peut-être la présence d'une cour desservant les pièces que nous avons dégagées sur les côtés nord et est.

Fig. 17 - Vue de détail du seuil de la pièce au sarcophage, et du sol contemporain SL 4010. 


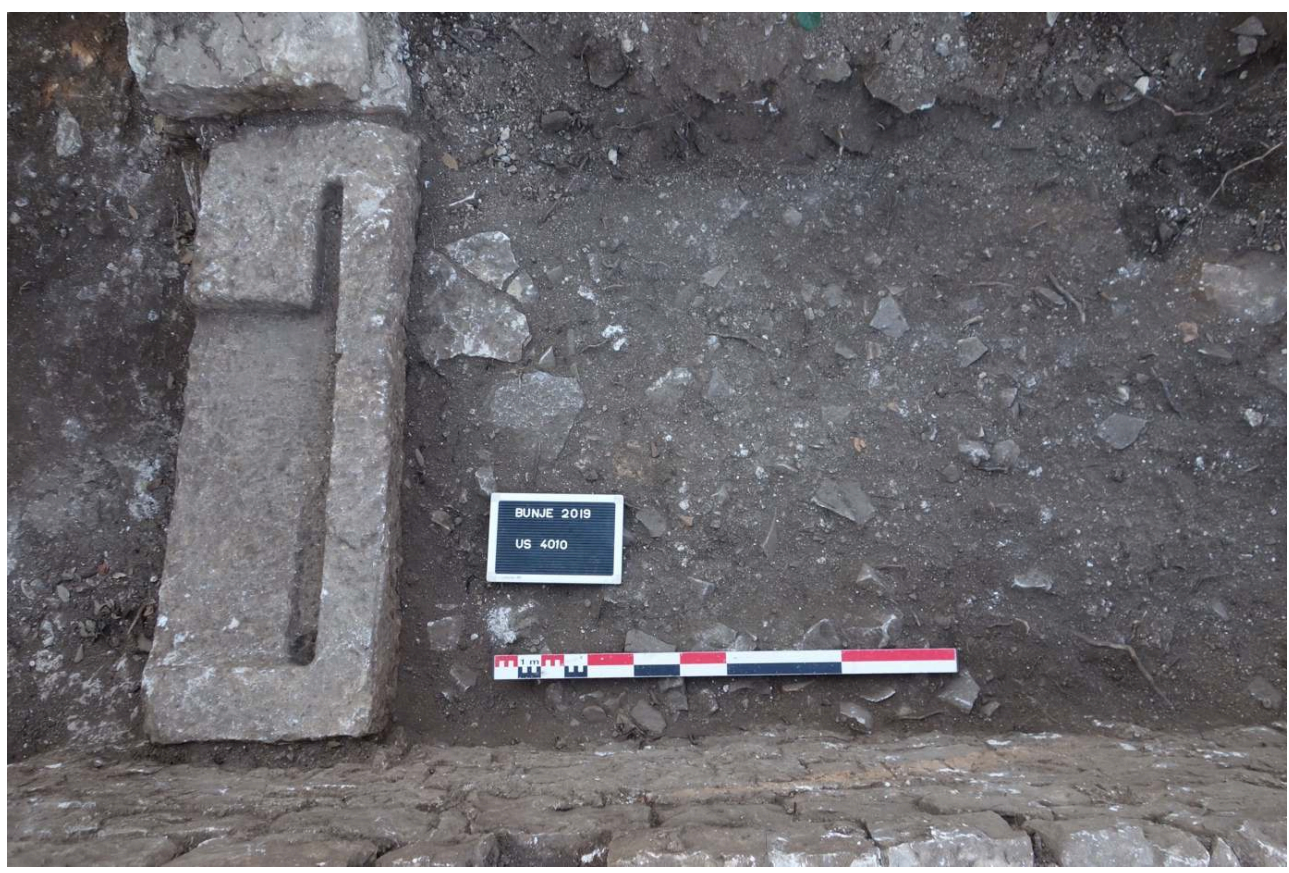

Photo E. Botte.

Fig. 18 - Vue depuis l'ouest de la pièce au sarcophage (secteur 3 ) et de son mur MR 3014 coupé pour faire passer le sarcophage.

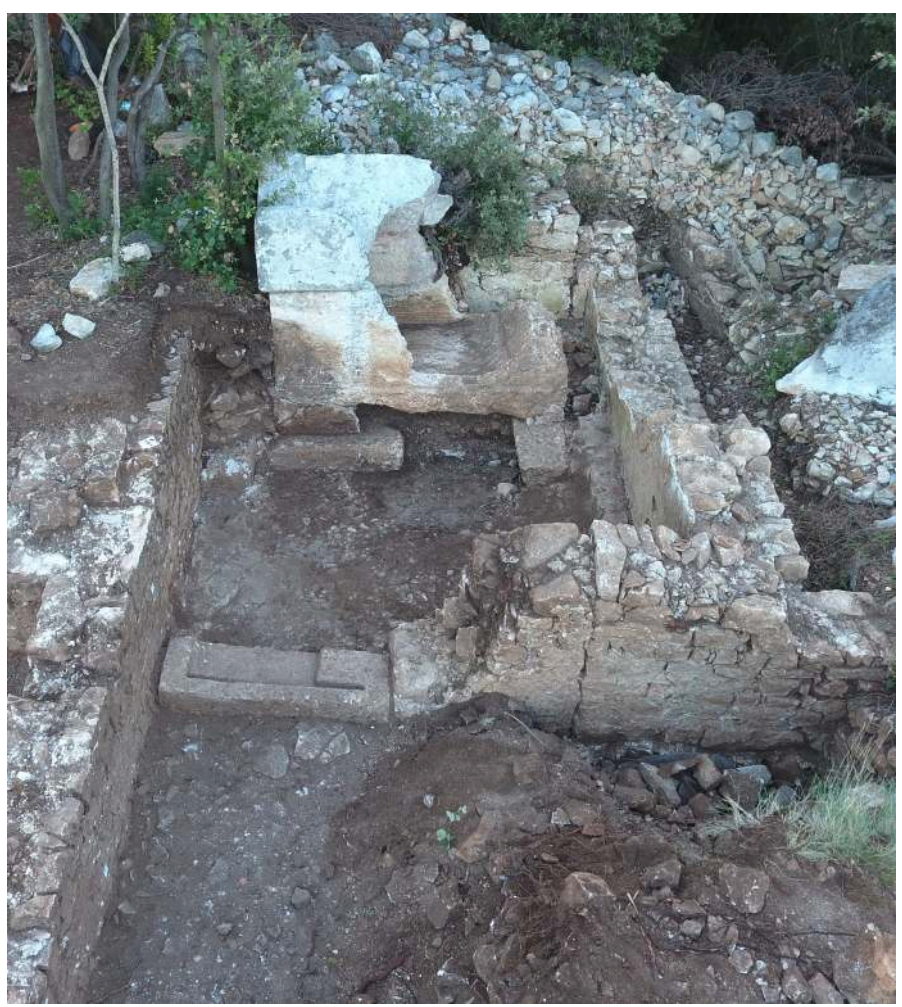

Photo E. Botte.

19 Enfin, une structure bâtie (BS 4005) est apparue sous l'angle formé par les murs MR 4022 et 4032 (fig. 19). De forme semi-circulaire, elle est construite en pierre calcaire 
et revêtue à l'intérieur d'un béton de tuileau. Elle mesure 1,20 ×1,50 m, et est conservée sur $1 \mathrm{~m}$ de hauteur. Le fond de cette structure est lui aussi tapissé de béton de tuileau. Notre première hypothèse était qu'il pouvait s'agir d'un puits, mais la présence du fond en béton de tuileau nous oriente clairement vers un bassin dont la présence à cet emplacement et la fonction ne sont pas encore déterminées. Il faut espérer que les dégagements de la prochaine campagne puissent nous apporter plus d'éléments de réponse, bien que sa présence nous permette d'ores et déjà de comprendre qu'il y avait dans cette terrasse des structures antérieures à son aménagement avec les murs aujourd'hui visibles.

Fig. 19 - Vue par drone du bassin BS 4005, sous les murs MR 4022 et 4032.

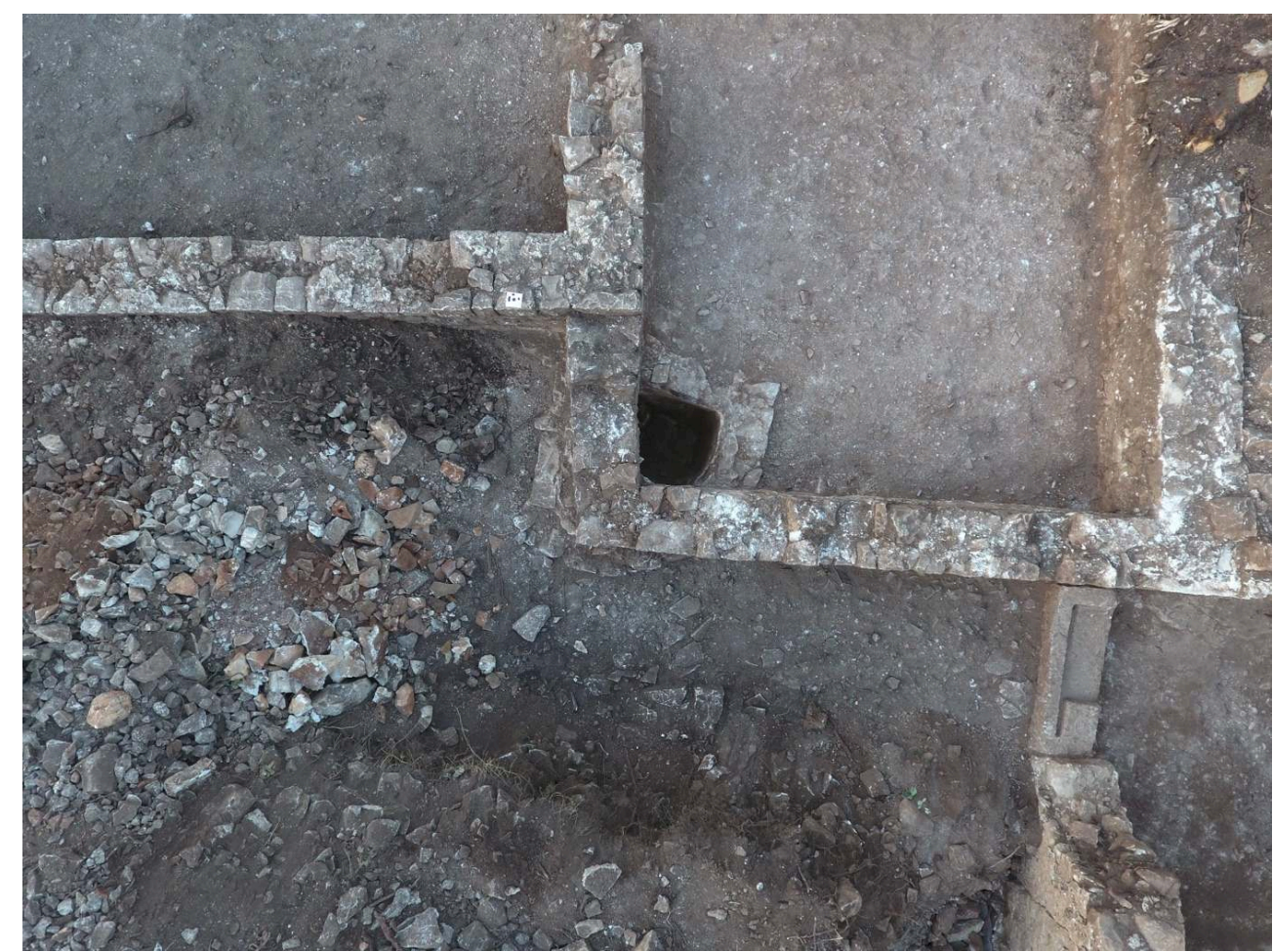

Photo E. Botte.

Ce bassin était comblé par un important remblai comportant beaucoup de céramiques, mais malheureusement aucune forme n'a pu être déterminée car il n'y avait aucun fragment de bord ou de fond caractéristique.

\section{Synthèse et programme des prochaines campagnes (fig. 20-21)}

Fig. 20 - Vue par drone de la villa de Bunje à la fin de la campagne de fouilles. 


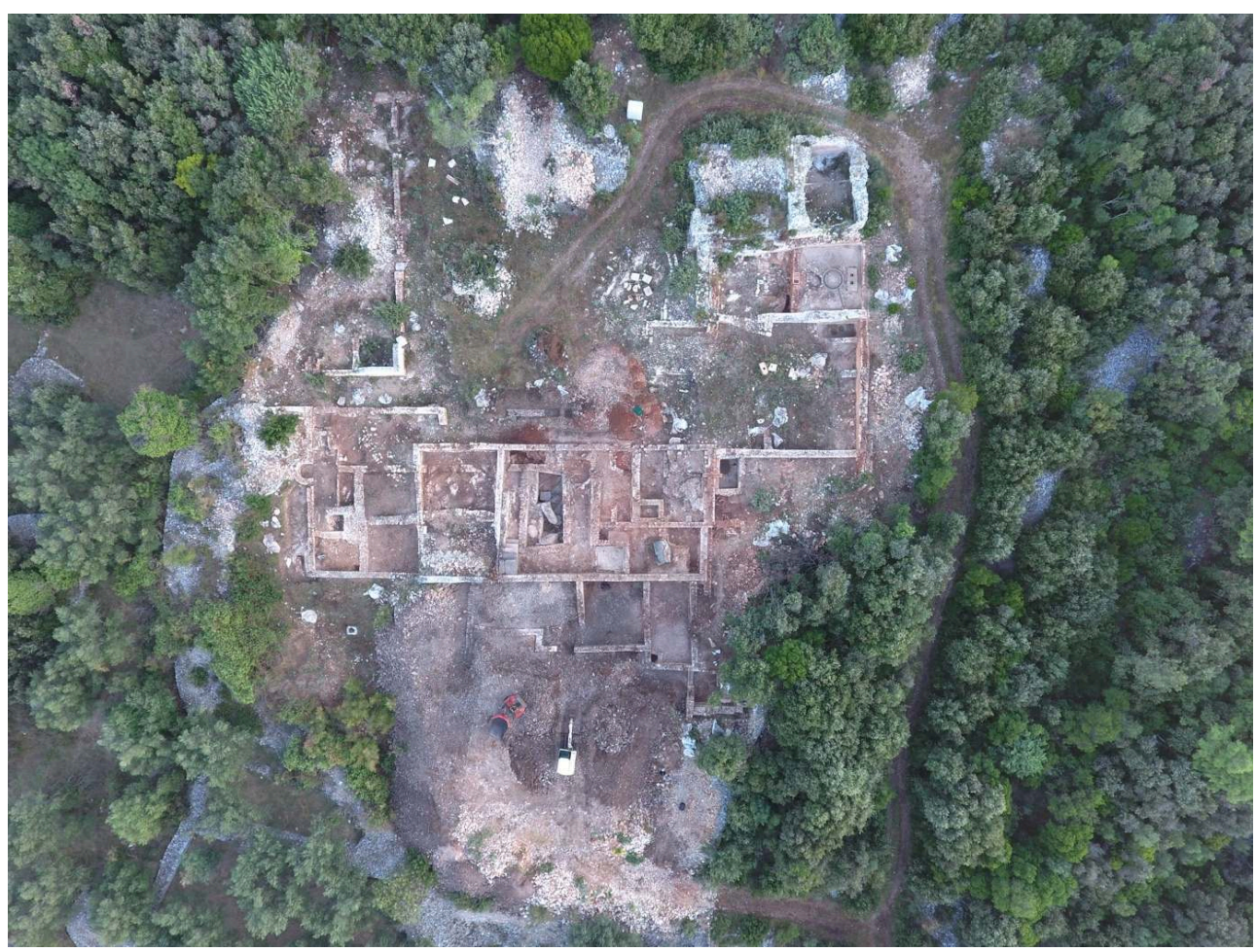

Photo E. Botte.

Fig. 21 - Plan de la villa à la fin de la campagne.

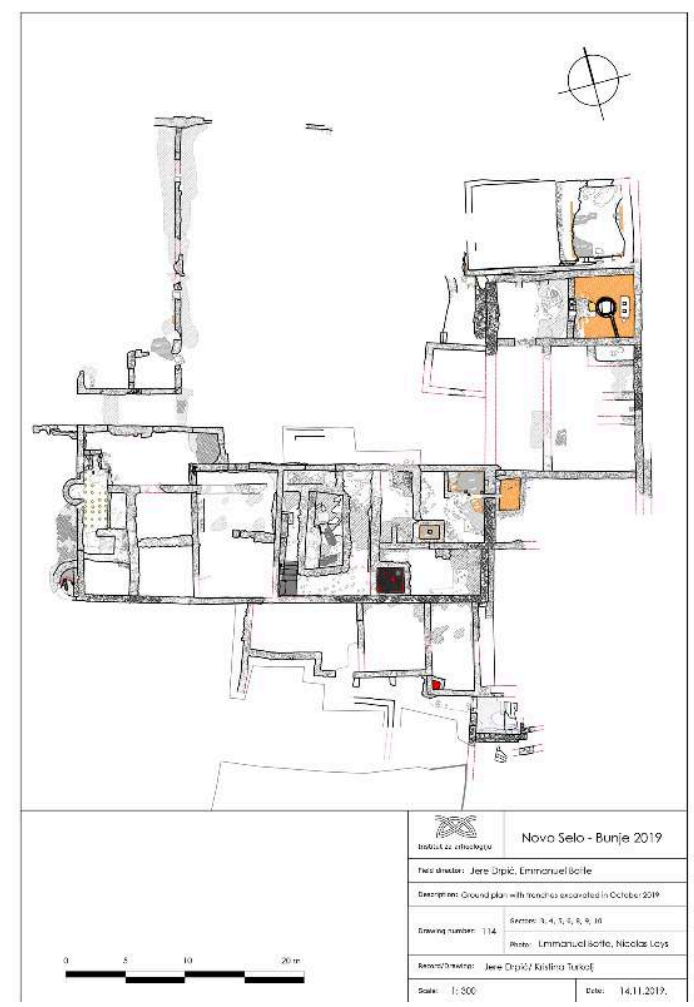

Plan J. Drpič. 
Au terme de cette campagne, nous estimons avoir rempli la plupart des objectifs que nous nous étions fixés au début de ce programme en 2016. La fouille de la villa de Bunje sur l'île de Brač constituait la première étape d'un projet à grande échelle géographique, dans lequel celle-ci devait nous permettre d'évaluer la faisabilité de notre projet. L'idée était en effet d'observer les modalités d'occupation du territoire au lendemain de sa conquête par les Romains et la fondation des premières colonies dans la seconde moitié $\mathrm{du} \mathrm{I}^{\mathrm{er}}$ siècle avant notre ère. Les résultats apportés par cette campagne de fouille nous ont permis de comprendre que sur le site de Bunje est d'abord bâtie une ferme, probablement autour du milieu du $\mathrm{I}^{\mathrm{er}}$ siècle de notre ère. Cet écart chronologique entre la fondation des colonies et l'installation du site n'est pas surprenant outre mesure. En effet l'île de Brač n'est pas la plus accueillante des îles dalmates en ce qui concerne son potentiel agricole. Face à des rivales comme Hvar, déjà occupée par les Grecs bien avant les Romains, Brač représentait surtout une immense carrière qui a permis d'y puiser les blocs nécessaires à la construction de cités comme Salona et Split. Ce n'est vraisemblablement qu'après une à deux générations de présence romaine que l'on peut observer la décision d'installer des villae également sur cette île. Néanmoins, la « surface agricole utile » de l'île est tellement faible par rapport à sa superficie (près de $400 \mathrm{~km}^{2}$ ), que le nombre de villae romaines l'est tout autant.

À la ferme au plan rectangulaire et aux dimensions modestes, mais dans laquelle un pressoir est déjà présent, très vraisemblablement pour la production de vin, tandis que des oliviers ont déjà dû être plantés par cette première génération de colons, succède dans le second tiers du $\mathrm{II}^{\mathrm{e}}$ siècle la villa telle que nous la voyons aujourd'hui dans son ensemble. Son plan est sans grande surprise celui de la villa romaine classique : de part et d'autre d'une cour centrale sont répartis des espaces de production (pars rustica) et résidentiels (pars urbana) comprenant des thermes et des espaces de vie. Il faut rester prudent car l'étude du mobilier archéologique n'est pas encore achevée, mais il semble qu'il y ait un abandon de la villa durant le III siècle, sans que l'on puisse être plus précis à ce stade de l'étude. Il est alors difficile de déterminer quand commence cet abandon. Il est en revanche établi qu'il prend fin dans le second tiers du IV siècle puisque l'on observe de grands remaniements, notamment dans le secteur de production avec l'installation d'un grand pressoir et le remaniement d'un autre. Nous ne disposons pas à ce stade de l'étude d'informations sûres pour déterminer si la terrasse inférieure est bâtie dès l'aménagement de la villa du II ${ }^{\mathrm{e}}$ siècle ou si cela intervient dans celle du IV siècle.

Le site est ainsi occupé jusqu'au $\mathrm{V}^{\mathrm{e}}$ siècle de notre ère, comme l'atteste la présence de deux sarcophages monumentaux en calcaire, typiques de cette période. Il semble enfin qu'il est voué à la récupération des matériaux et des pierres travaillées à partir de la fin $\mathrm{du} \mathrm{V}^{\mathrm{e}}$ et au début du $\mathrm{VI}^{\mathrm{e}}$ siècle.

\section{Perspectives du programme}

Nous envisageons pour la suite du programme une série de vérifications nécessaires en vue de la publication de la fouille. Il faut notamment que nous comprenions l'organisation de la terrasse inférieure, et la circulation avec celle supérieure. D'autre part, il nous faut encore travailler sur l'alimentation en eau de la villa. Nous opérerons donc un nettoyage et un relevé du réservoir d'eau douce creusé contre le flanc sud de la montagne au pied de laquelle est bâtie la villa. 


\section{BIBLIOGRAPHIE}

Botte - Bertrand - Jelinčić 2016 = E. Botte, A. Bertrand, K. Jelinčić, Bunje (Novo Selo, Croatie), dans Chronique des activités archéologiques de l'École française de Rome, 2016. DOI : 10.4000/cefr.1519

Botte - Jelinčić 2017 = E. Botte, K. Jelinčić, Bunje (Novo Selo, Croatie), dans Chronique des activités archéologiques de l'École française de Rome, 2017. DOI : 10.4000/cefr.1723

Botte et al. 2019 = E. Botte, A. Bertrand, K. Jelinčić, N. Leys, A. Boisson, Bunje (Novo Selo, Croatie), campagnes 2017-2018, dans Chronique des activités archéologiques de l'École française de Rome, 2019. DOI : $10.4000 /$ cefr. 2419

Jelinčić $2012=\mathrm{K}$. Jelinčić, Izvješće arheološkom rekognosciranju i snimanju lokaliteta Novo Selo Bunje (18.-19. travnja 2012.), Zagreb, 2012.

\section{NOTES}

1. Pour un résumé des résultats des campagnes précédentes, voir Botte - Bertrand Jelinčić 2016 ; Botte - Jelinčić 2017 ; Botte et al. 2019.

2. Jelinčić 2012.

\section{INDEX}

institutions École française de Rome, Ministère de l'Europe et des Affaires étrangères, Institut Archéologique de Zagreb, Centre Camille Jullian, Université Paris-Est Marne, mairie de Selca Index géographique : Bunje

Mots-clés : Dalmatie, villa romaine, vin, huile, Antiquité

\section{AUTEURS}

\section{EMMANUEL BOTTE}

Aix Marseille Univ, CNRS, CCJ, Aix-en-Provence, France

\section{AUDREY BERTRAND}

Univ. Paris-Est Marne

JERE DRPIĆ

Institut archéologique de Zagreb

\section{KRISTINA JELINČIĆ}

Institut archéologique de Zagreb 


\section{BASTIEN LEMAIRE}

Univ. Montpellier 3

NICOLAS LEYS

Sorbonne Université

FLORIAN HUVET

Mosaïques Archéologie

ANTOINE BOISSON

INRAP 